\title{
Open Rectangle-of-Influence Drawings of Non-triangulated Planar Graphs
}

\author{
Soroush Alamdari and Therese Biedl \\ Cheriton School of Computer Science, University of Waterloo, Waterloo, Canada \\ \{s26hosse, biedl\} @uwaterloo.ca
}

\begin{abstract}
A straight line drawing of a graph is an open weak rectangle-of-influence ( $R I)$ drawing if there is no vertex in the relative interior of the axis parallel rectangle induced by the end points of each edge. Despite recent interest of the graph drawing community in rectangle-of-influence drawings, no algorithm is known to test whether a graph has a planar open weak RI-drawing.

In a recent paper, we showed how to test, for inner-triangulated planar graphs, whether they have a planar open weak RI-drawing with a non-aligned frame, i.e., the graph obtained from removing the interior of every filled triangle is drawn such that no two vertices have the same coordinate. In this paper, we generalize this to all planar graphs with a fixed planar embedding, even if some interior faces are not triangles. On the other hand, we show that if the planar embedding is not fixed, then deciding if a given planar graph has an open weak RI-drawing is NP-complete. NP-completeness holds even for open weak RI-drawings with non-aligned frames.
\end{abstract}

Keywords: Proximity drawings, Rectangle-of-Influence drawings, Angular labeling.

\section{Introduction}

The rectangle-of-influence (RI for short) drawability problem was introduced by Liotta et al. [8]. In a strong RI drawing of a graph, there is an edge between two vertices of the graph if and only if there is no other vertex in the axis-parallel rectangle defined by the two ends of every edge. There are two variants of RI-drawings: In a closed RI-drawing, the rectangle required to be empty is closed, whereas in an open RI-drawing, only the relative interior of the rectangle is required to be empty (see Figure 4(c).) Liotta et al. [8] gave a characterization of cycles, wheels, outer-planar graphs, and triangle-free graphs that are strong open RI drawable. For strong closed RI drawable graphs, they presented complete characterization of cycles, wheels and trees.

Biedl et al. [3] were the first to study weak RI drawings. This means that graphs are drawn such that for any edge the corresponding axis-aligned rectangle is empty, but for all such empty rectangles the edge is not necessarily present. They proved that a plane graph has a planar weak closed RI drawing on $n \times n$ grid if and only if it has no filled triangle. Recently, Sadasivam and Zhang [13] improved this grid size to $(n-3) \times(n-3)$ and showed that this is tight. 
For open RI drawings, better bounds for the size of the drawing are known. Miura and Nishizeki [11] and Zhang and Vaidya [16] provide small weak open RI drawings for some classes of graphs that have no filled triangles.

But no necessary and sufficient conditions are known for the existence of (weak planar) open RI-drawings, even for inner triangulated graphs. This study was initiated by Miura, Matsuno and Nishizeki [10]. They aimed to develop necessary and sufficient conditions for existence of a weak open RI-drawing for inner triangulated graphs, but did not succeed. They assumed extra conditions on the drawing of the frame graph, which is the graph obtained by removing the inside of every filled triangle (see Figure 2 (a,b).) They only considered weak open RI-drawings with oblique frame in which no edges of the frame graph are drawn horizontally or vertically. For this case they gave one set of conditions that are clearly necessary, and showed that adding one condition made them sufficient.

In our previous paper [1], we studied weak open RI-drawings with non-aligned frame, which means that no two vertices of the frame graph have the same $x$-coordinate or the same $y$-coordinate. We presented necessary and sufficient conditions to test if an inner triangulated graph has an RI-drawing with non-aligned frame. We also presented an algorithm to construct such a drawing if one exists. As our first major result, in Section 5 we generalize this algorithm so that it works for plane graphs even if not all inner faces are triangles.

Liotta, in the Chapter "proximity drawings" of [14] points out that "it would be interesting to characterize which planar graphs have a weak open rectangle of influence drawing". In Section 6, we show that likely there is no easily verifiable characterization of such graphs, by proving NP-completeness of the problem of deciding if a planar graph has an open planar RI-drawing. To our knowledge, this is the first hardness result regarding RI drawings. The proof is based on proof of hardness of finding orthogonal drawings of planar graphs by Garg and Tamassia [7].

\section{Preliminaries}

We presume familiarity with planar graphs and planar drawing notations; see for example [12]. In particular, in this paper $G$ is always a planar graph. We use the term plane graph if we assume that the planar embedding (clockwise order of vertices around edges) and the outer-face of $G$ have been fixed. Given a plane graph $G$, the dual graph is obtained by creating a vertex $v_{f}$ for every face $f$, and adding an edge $\left(v_{f}, v_{g}\right)$ whenever faces $f$ and $g$ share an edge.

A straight-line drawing of $G$ is called an orthogonal drawing if all edges are axis aligned. (We do not allow any bends in such a drawing.) We will be using orthogonal drawings in both our major results, showing a strong connection between RI-drawings and special cases of orthogonal drawings. A rectangle-contact dual drawing of $G$ represents the vertices of $G$ by a set of non-overlapping rectangular faces, where two rectangles share part of a side if and only if there is an edge between their corresponding vertices in $G$. A rectangular drawing is an orthogonal drawing in which all faces have rectangular boundaries; it is called inner-rectangular if all faces but the outer-face are rectangular. An inner-rectangular drawing of $G$ is a rectangle-contact drawing of 
the dual of $G$ minus the outer-face vertex. But rectangle-contact drawings may have "holes" between the rectangles representing vertices, a fact that we will exploit in our algorithm.

\section{Axis-Count Labelings}

A crucial insight for testing the existence of RI-drawings (and also orthogonal drawings) is to express such drawings via a labeling of the (graph-theoretic) angles, i.e., the place where two edges are consecutive at a vertex. We will briefly give here a new way of assigning labels to angles of a graph drawing such that it includes the labeling methods for RI drawings [110] as well as orthogonal drawings (e.g. [2[14]).

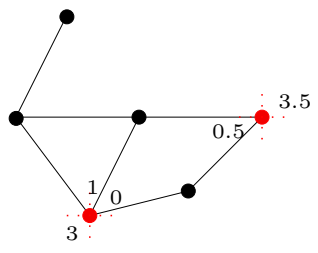

Fig. 1. An open RI drawing with some of the AC labels

An Axis-Count labeling (AC for short) for a straight-line drawing of a graph is a labeling defined as follows: Let $\Gamma_{G}$ be a straight-line drawing of a graph $G$. To each angle $\alpha$ of $G$ we assign a label $\ell(\alpha) \in\{0, .5,1,1.5,2,2.5,3,3.5,4\}$ that counts how many coordinate axes are covered by this angle (see Fig. 10. More precisely, let $0 \leq a \leq 2$ be the number of axis-aligned edges of $\alpha$ in $\Gamma_{G}$. Let $b$ be the number of coordinate axis in the interior of $\alpha$. Then $\ell(\alpha)=\frac{a}{2}+b$.

The following is easy to show.

Lemma 1. Any AC labeling of a connected graph has the following properties:

a) The sum of labels around each vertex is 4 .

b) The sum of labels on an inner face with $k$ angles is $2 k-4$.

c) The sum of labels on the outer face with $k$ angles is $2 k+4$.

AC labelings generalize labelings for orthogonal drawings. The following result was proved by Miura et al. [9] and also follows from a prior result on orthogonal shapes by Tamassia [15].

Lemma 2. [9] An angle-labeling of a plane connected graph $G$ with labels in $\{1,2,3,4\}$ can be realized as an orthogonal drawing such that each (graph-theoretic) angle of label $i$ has (geometric) angle of $i \pi / 2$, if the labeling satisfies

(a) For each vertex, the labels of incident angles sum to 4.

(b) The sum of the labels on an inner face $f$ is $2 k-4$, where $k$ is the number of angles on the face.

(c) The sum of the labels on the outer-face is $2 k+4$, where $k$ is the number of angles on the outer-face.

We call such a labeling OD-admissible. Note that by Lemma 1 and Lemma 2 an AC labeling is OD-admissible if and only if all its labels are in $\{1,2,3,4\}$.

Angle-labelings were also studied for RI-drawings by Miura et al [10], and in our earlier paper [1]. In these papers, the studied RI-drawings were oblique (no horizontal or vertical edges), in which case $\ell(\alpha) \in\{0,1,2,3,4\}$ counts the number of coordinate axes contained in angle $\alpha$. We review some properties of these labelings. 
Lemma 3. [10] In any oblique RI-drawing of a graph, any triangular inner face has AC-labels $\{0,1,1\}$.

We say that two angles are adjacent if they share an edge and are on the same face. A chain $C=\left(\alpha_{0}, \alpha_{1}, \ldots, \alpha_{k-1}\right)$ of angles is a sequence of distinct adjacent angles along a face. We denote the number of angles in $C$ by $|C|$. Slightly abusing notation, we also sometimes use $C$ for the sequence of AC-labels that are assigned to the angles of $C$ (in some drawing that is clear from the context.)

Lemma 4. [1] Let $C$ be a chain of angles in a planar RI-drawing. Then $C$ does not contain 00 as a subsequence of its labels.

Lemma 5. [1] Let $C$ be a chain of angles in a planar non-aligned RI-drawing. Then $C$ does not contain $01^{+} 0$ as a subsequence of its labels. Note that $01^{+} 0$ is a sequence of ones bounded by zeros.

We need one new property for the NP-hardness in Section 6 , the proof is lengthy and involves heavy case analysis.

Lemma 6. Let $C$ be a chain of angles in a planar non-aligned RI-drawing of a 2connected graph. Then the sum of labels in $C$ is in the range $[|C|-3,3|C|+3]$.

\section{Graphs with Triangular Outer-Face}

Similar as in previous papers, we characterize the existence of RI-drawings by studying the frame of a graph, which is the graph obtained by removing the inside of filled triangles. To be able to re-insert the filled triangles, we hence need to study first existence of RI-drawings of plane graphs where the outer-face is a triangle. This was very simple if the graph is inner-triangulated (see [10]), but becomes more complicated for arbitrary planar graphs.

Lemma 7. Let $G$ be a plane graph with triangular outer face. Then one can compute in linear time all possible labelings of the outer face that could occur in an open RIdrawing of $G$ with oblique outer face. Also, given any such labeling of the outer-face, it can be completed to an RI-drawing of $G$ in linear time.

Proof. Presume we have an open RI-drawing drawing of $G$ with oblique outer-face. Since the outer-face is a triangle, its angles must have labels $\{3,3,4\}$; we only must test which of the outer-face vertices could have label 3. Since the three rectangles defined by the outer-face edges must be empty except at the boundary, this leaves only one horizontal line and one vertical line where interior vertices of $G$ could be placed. The graph between the two vertices with label 3 hence must be drawable on two lines, a condition that can be tested in linear time [6]. Moreover, the two outer-face vertices with label 3 must be at the end of these two lines. We can modify the algorithm by Cornelsen et al. so that it takes these restrictions on the outer-face vertices into account and hence test whether a given labeling of the outer-face can be realized. 
Note that the set of feasible labelings can be described succinctly by saying which vertices on the outer-face must be labeled 3 (this is possible regardless of which subset of the three possible labelings is feasible.) In the following, we will instead express this by fixing which inner labels of the triangular face corresponding to a filled triangle must be 1 ; this is the same since labels at vertices must sum to 4 .

\section{Testing RI-Drawability of Plane Graphs}

In a previous paper [1], we presented an algorithm to obtain RI-drawings of inner triangulated graphs with non-aligned frame. Here with simple modifications we generalize the algorithm to work for any plane graphs rather than only inner triangulated plane graphs.

Let $G$ be a plane graph. Let $\mathcal{F}$ be the frame graph of $G$. In this section we give a constructive algorithm to decide whether $G$ admits an open RI drawing such that $\mathcal{F}$ is drawn non-aligned. Here, we assume the outer face is not a triangle, as we already handled that case in the previous section. We also presume that for each filled triangle we have already computed (as discussed after Lemma 7) the set of angles of the frame graph that are forced to be 1 if we want to have an RI-drawing of the graph inside the filled triangle. Let $A$ be the set of all angles that are forced to be 1 .

Definition 1. A labeling of the angles of the frame graph $\mathcal{F}$ with labels in $\{0,1,2,3,4\}$ is RI-admissible if (a) the labels at every vertex sum to $4,(b)$ the labels at each inner face with $k$ angles sums to $2 k-4$, and every angle in $A$ is labeled 1 , where $A$ is the set of restrictions implied by the filled triangles, and (c) there is no chain of angles with labels of the form $01^{*} 0$.

(This definition is almost identical to the one in [1], except that condition (b) was generalized to arbitrary faces.) Our first main result is:

Theorem 1. A plane graph $G$ has a planar weak open RI-drawing with non-aligned frame if and only if the frame graph $\mathcal{F}$ has an RI-admissible labeling. Also, such drawing can be constructed in $O\left(n^{1.5}\right)$ if it exists.

The necessity of conditions (a) and (b) follows directly from Lemma 1 and condition (c) is necessary by Lemmas 5 and 4 . Sufficiency is proved by giving an algorithm that finds a drawing for any RI-admissible labeling. The algorithm is similar to the algorithm presented in [1]. We briefly outline this algorithm to keep the paper somewhat readable without requiring [1], but we focus on the changes. The algorithm works as follows:

(i) Compute the frame graph $\mathcal{F}$ (see Fig. 2(b)).

(ii) For every triangle $T$ of $\mathcal{F}$ that was filled in $G$, compute whether the interior of $T$ is realizable in an open RI-drawing as in Lemma 7 If this fails for any triangle, then $G$ has no open RI-drawing. Else, let $A$ be the set of angles of $\mathcal{F}$ that must have label 1 (see Fig. 2(b)).

(iii) For any face $f$ of $\mathcal{F}$ that has degree $k>3$, add a vertex $v_{f}$ inside, and connect it with a triple edge to every vertex of $f$ so that $v_{f}$ has degree $3 k$.

[In [1], we applied this operation only to the outer-face.]

Let $D$ be the dual graph of the resulting graph. See Fig. 2(c)). 


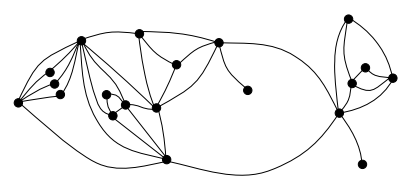

(a)

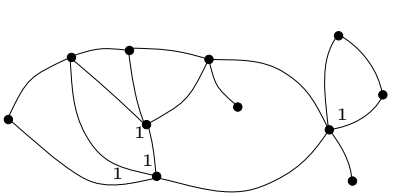

(b)

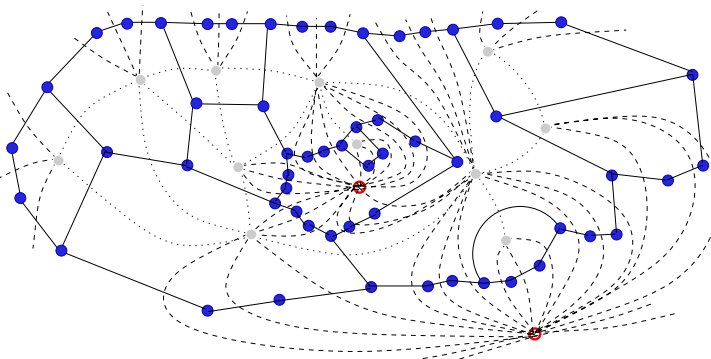

(c)

Fig. 2. (a) Graph $G$, (b) its frame graph $\mathcal{F}$ with forced AC labels, (c) the dual-like graph $D$ (solid)

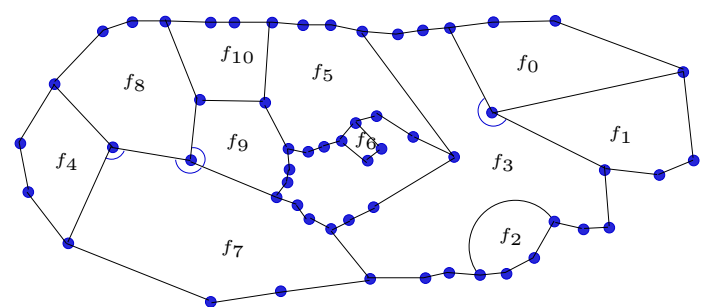

(a)

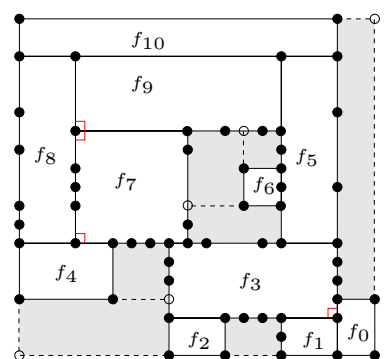

(b)

Fig. 3. (a) Graph $D$, (b) a rectangle-contact dual drawing expanded to a rectangular drawing by adding dashed edges and shaded rectangles

(iv) Find a drawing $\Gamma_{D}$ of $D$ that is a rectangle-contact drawing of $\mathcal{F}$ and respects $A$ in some sense. See Fig. 3 If there is none, stop: $G$ does not have an RI-drawing with non-aligned frame.

[In [1], we used an inner-rectangular drawing in place of the rectangle-contact drawing.]

(v) Expand $\Gamma_{D}$ into a rectangular drawing $\Gamma_{D^{\prime}}$ of a super-graph $D^{\prime}$ of $D$. Do this by adding edges and vertices to the graph, without changing the rectangular faces, so that $\Gamma_{D^{\prime}}$ also respects $A$ (see Fig. 3 (b)).

(vi) Construct the dual graph $\mathcal{D}\left(D^{\prime}\right)$ and then remove the outer face vertex. The resulting inner triangulated graph $\mathcal{F}^{\prime}$ is a super-graph of the frame-graph $\mathcal{F}$ (see Fig. $4(a)$ ).

(vii) The rectangular drawing of $D^{\prime}$ defines an RI-admissible labeling of $\mathcal{F}^{\prime}$ that has special properties.

(viii) Using this RI-admissible labeling, create a non-aligned RI-drawing of $\mathcal{F}^{\prime}$ using a variant of the algorithm presented in [10]. See Fig. 4(b).

(ix) Then insert the filled triangles to obtain an open RI-drawing with non-aligned frame of a super-graph $G^{\prime}$ of $G$.

(x) Remove the vertices of $V_{G^{\prime}} \backslash V_{G}$ from the drawing (see Fig.4(c)). 


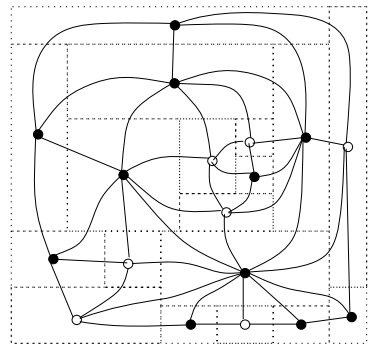

(a)

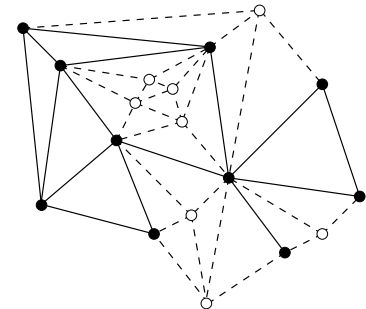

(b)

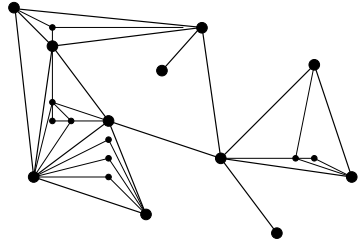

(c)

Fig. 4. (a) The drawing $\Gamma_{D^{\prime}}$ (dotted edges) and the graph $\mathcal{F}^{\prime}$, (b) the RI-drawing of $\mathcal{F}^{\prime}$ obtained by the labeling (c) and the RI-drawing of $G$ obtained by putting the inside of filled triangles back in and removing the dummy vertices

Steps (i), (iii) and (x) are straightforward. Steps (vi)-(ix) are exactly as in [1]. Step (v) can be done as in [1] by applying the routine to all non-triangular faces. Steps (ii) and (ix) follow from Lemma 7 So the only major difference is in step (v). First, we must prove correctness: If $G$ has an open RI-drawing with non-aligned frame, then $\mathcal{F}$ has a rectangle-contact drawing. This uses many of the ideas in [1], but requires some attention to details. Secondly, we must show how to test existence of a rectangle-contact drawing. This is done by using Tamassia's network-flow approach [15] that computes an OD-admissible labeling and allows to impose the additional restrictions. An algorithm presented recently by Cornelsen and Karrenbauer [5] can be used to do this in $O\left(n^{1.5}\right)$ time, improving over the $O\left(n^{1.5} \log n\right)$ mentioned in [1]. This proves Theorem 1

\section{Hardness of Testing RI-Drawability of Planar Graphs}

No results exist for open planar RI-drawings if the planar embedding can be chosen. In this section, we show that testing the existence of such drawings is NP-complete. This is in contrast with closed planar RI drawings, for which Biedl et al. [4] present a polynomial time algorithm to find a closed RI-drawable embedding if one exists. The reduction presented here is based on the one by Garg and Tamassia [7] used for proving NP-hardness of finding orthogonal drawings of a given planar graph.

Let $P$ be an undirected graph with a finite capacity set $s(e) \subseteq \mathbb{N}$ assigned to each edge $e \in E(P)$. An integer switch-flow is an assignment of direction and flow $f(e) \in \mathbb{N}$ to each edge $e \in E(P)$ such that $f(e) \in s(e)$ for any $e \in E$ and such that the balancecondition holds: For every vertex $v$ the sum of the incoming flow is equal to the sum of the outgoing flow. Garg and Tamassia study this problem, and prove, with a reduction from NOT-ALL-EQUAL-3-SAT, that it is NP-hard even in the following restriction: 1

1. $P$ is a planar triconnected graph.

2. Any edge $e$ has either only one possible capacity, $s(e)=\left\{c_{e}\right\}$ (we call these fixed edges), or it has an interval of capacities $s(e)=\left\{0,1, \ldots, c_{e}\right\}$ (we call these flexible edges).

\footnotetext{
${ }^{1}$ Their constants were slightly different from what we list below, but these constants can be achieved by modifying their construction slightly.
} 
3. For any fixed edge with capacity $\left\{c_{e}\right\}$ or flexible edge with capacity set $\left\{0, \ldots, c_{e}\right\}$, we know $c_{e}$ is larger than $\theta=4 \gamma+4$, where $\gamma$ is an upper bound on the degree of faces of $P$.

Theorem 2. Testing whether a planar graph has an open planar RI-drawing is NPcomplete.

Proof. It is easy to see that the problem is in NP, for if $G$ has an open planar RI-drawing, then it also has one in an $n \times n$-grid (any planar RI-drawing can be deformed arbitrarily without creating crossings as long as the relative $x$-order and $y$-order of vertices remains intact.)

To prove NP-hardness, we reduce from the special case of the integer switch-flow problem explained above. Presume we are given such an instance $\mathcal{I}$ with planar graph $P$. We proceed as follows:

(i) Define a graph $G_{O D}$ in such a way that $G_{O D}$ has an orthogonal drawing if $\mathcal{I}$ has a solution.

(ii) Based on $G_{O D}$, define a graph $G_{R I}$ such that $G_{O D}$ is a subgraph of $G_{R I}$.

(iii) Show that if $G_{O D}$ admits an orthogonal drawing then $G_{R I}$ admits an open RI drawing. In fact, the drawing has a non-aligned frame.

(iv) Finally, show that if $G_{R I}$ admits an open RI drawing, then $\mathcal{I}$ has a solution.

Step (i) is exactly as in [7] except that we use slightly enlarged gadgets. Steps (ii), (iii), (iv) define our contribution.

Tendrils and Wiggles: To define the graphs $G_{R I}$ and $G_{O D}$, we require some definitions and intermediate gadgets first. A rectilinear $i$-wiggle $M$, for a positive integer $i$, is a path of length $8 i+1$. The two ends of a rectilinear $i$-wiggle $M$ are its designated vertices $s_{M}, t_{M}$.

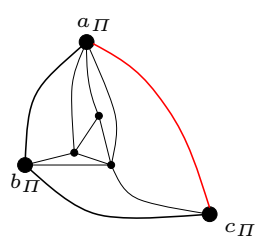

Fig. 5. The graph $\Pi$

The $R I$-wiggle $W$ is constructed from the rectilinear $i$-wiggle $M$ by replacing each edge $(u, v)$ of the rectilinear $i$-wiggle where $u, v \notin\left\{s_{M}, t_{M}\right\}$ with an instance of the graph $\Pi$ depicted in Fig. [5 such that $u$ and $v$ correspond to $b_{\Pi}$ and $a_{\Pi}$, respectively, where $u$ is closer to $s_{M}$ in $M$ than $v$ (see Fig. 6). The initial rectilinear $i$-wiggle inside the RI $i$-wiggle $W$ is called the backbone of $W$.

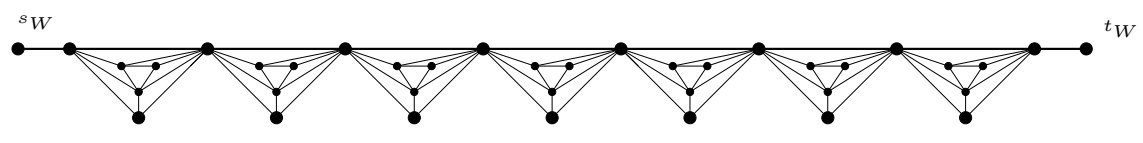

Fig. 6. An RI $i$-wiggle

A rectilinear $i$-tendri $2 R$ is an undirected graph with $16 i+14$-cycles and two designated vertices $s_{R}$ and $t_{R}$ as shown in Fig.7(a).

${ }^{2}$ Our rectilinear $i$-wiggle is equivalent to the $2 i$-wiggle defined in [7], and our rectilinear $i$ tendril is equivalent to the $2 i$-tendril defined in [7]. 


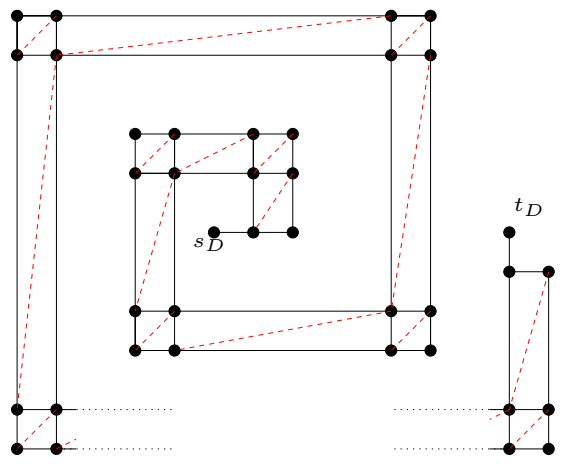

(a)

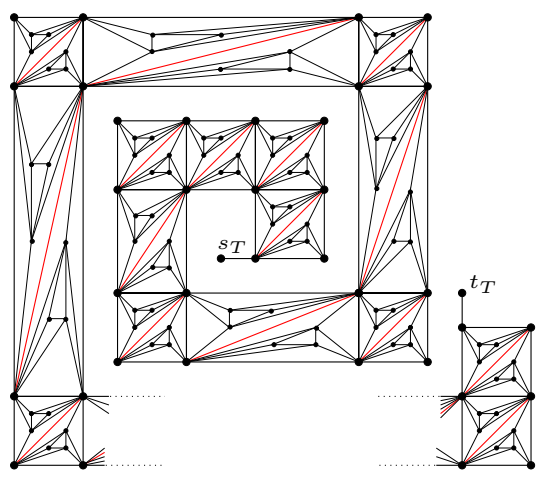

(b)

Fig. 7. (a) A rectilinear $i$-tendril (solid) and the diagonals (dashed) added for the diagonal $i$ tendril, (b) an RI $i$-tendril obtained by adding instances of graph $\Pi$

Lemma 8. 77 Any rectilinear $i$-tendril $R$ has exactly one planar embedding with both designated vertices in the outer face.

The diagonal $i$-tendril is constructed from the rectilinear $i$-tendril by adding a diagonal edge in each four-cycle as depicted in Fig.7(a). The $R I$ - $i$-tendril is constructed from the diagonal $i$-tendril by replacing each triangle by the graph $\Pi$ such that the edge $\left(a_{\Pi}, c_{\Pi}\right)$ corresponds to the diagonal edge, and the planar embedding of $\Pi$ is maintained, i.e., the counter clockwise order is $\left\{a_{\Pi}, b_{\Pi}, c_{\Pi}\right\}$ along the triangle. (See Fig. 7 b).) By Lemma 8 and since $\Pi$ is triconnected, we have:

Lemma 9. Any RI $i$-tendril $T$ has exactly one planar embedding with both designated vertices in the outer face.

Now we give details for steps (i)-(iv). Step (i) was done in [7], but we review it briefly since step (ii) builds on it. Let $P$ be the planar graph of the integer switch-flow problem and let $D$ be its dual graph. To construct the graph $G_{O D}$, first replace each vertex of $D$ of degree $d>4$ by a binary tree with $d$ leaves so that each of its neighbors in $D$ is adjacent with one of the leafs of the binary tree. Let $D^{\prime}$ be the resulting graph. Let $e$ be an edge of $D^{\prime}$ that also is an edge of $D$, and let $e^{\prime}$ be the edge in $P$ for which $e$ is the dual edge.

- If $e^{\prime}$ is flexible, say $s\left(e^{\prime}\right)=\{0,1, \ldots, c\}$ for some $c$, then substitute $e$ with a rectilinear $c$-wiggle.

- Else $e^{\prime}$ is fixed, say $s\left(e^{\prime}\right)=\{c\}$ for some $c$. Substitute $e$ with a rectilinear $c$-tendril.

Lemma 10. [7] All the embeddings of $G_{O D}$ are obtained by choosing one of the two possible flips for each rectilinear tendril.

See [7] for a proof that if $P$ has a solution, then $G_{O D}$ has an orthogonal drawing. In a nutshell, a solution to the switch-flow problem assigns a direction to each fixed edge, which determine which of the two possible flips of the tendril at this edge should be 
used. This determines the planar embedding of $G_{O D}$ to be used for the orthogonal drawing, and it is then not hard to find an OD-admissible labeling since most of the graph has a unique one.

Now we come to step (ii). $G_{R I}$ is constructed in the same way as $G_{O D}$, with the difference that we use RI tendrils and RI wiggles instead of rectilinear tendrils and rectilinear wiggles, respectively. Note that each RI tendril (resp. wiggle) contains a rectilinear tendril (resp. wiggle) as a subgraph, so $G_{O D}$ is a subgraph of $G_{R I}$. Since tendrils have a unique planar embedding with designated vertices on the outer-face, we have by Lemma 10 .

Corollary 1. All the embeddings of $G_{R I}$ are obtained by choosing one of the two possible flips for each RI tendril and for each copy of $\Pi$ in each RI wiggle.

We must prove that if $G_{O D}$ has an orthogonal drawing $\Gamma_{O D}$, then $G_{R I}$ has an open RI drawing with non-aligned frame. Presume we have such an orthogonal drawing $\Gamma_{O D}$. Choose the planar embedding for $G_{R I}$ such that the induced planar embedding of $G_{O D}$ is as in $\Gamma_{O D}$. This fixes the flips for all RI-tendrils. For each RI-wiggle, we flip the instances of $\Pi$ in such a way that we can label the frame-graph of the RI-wiggle without violating RI-admissibility; this is not difficult but requires a small case analysis.By transferring all other angular labels from $\Gamma_{O D}$, we obtain an angular labeling of the frame graph of $G_{R I}$ that is easily seen to be RI-admissible. Hence $G_{R I}$ has a planar open RI-drawing with non-aligned frame, which finishes step (iii).

The final (and hardest) part of the NP-hardness is step (iv), where we must construct a valid solution for the integer switch-flow of $P$ from an open planar RI drawing $\Gamma_{R I}$ of $G_{R I}$. Recall that each edge $e$ of $P$ corresponds to either an RI-wiggle or an RI-tendril in $G_{R I}$ that replaces the dual edge of $e$. We read the direction for $e$ from the embedding that has been used for this tendril/wiggle, but need some observations to formalize this.

Recall that by Lemma 6 for any chain $C$ of angles the sum of the labels is in the range $[|C|-3,3|C|+3]$. It will be easier to speak instead of the contribution of $C$, which is the sum of the labels of $C$, minus $2|C|$, and which is therefore in the range $[-|C|-3,|C|+$ $3]$. The contribution of such a chain corresponds roughly to its turn-angle (divided by $\pi / 2$ ). Analyzing the structure of RI-wiggles and RI-tendrils, the following lemmas are easy to prove.

Lemma 11. Let $W$ be an RI $i$-wiggle in $G_{R I}$ adjacent to face $f$. Then the chain of angles at vertices of $W$ incident to $f$ has contribution in $[-8 i-4,8 i+4]$.

Lemma 12. Let $R$ be an $R I$-tendril in $G_{R I}$ adjacent to face $f_{1}$ and $f_{2}$. Then (after possible renaming of $f_{1}$ and $f_{2}$ ) the chain of angles at vertices of $R$ incident to $f_{1}$ has contribution in $[8 i-3,8 i+4]$ and the chain of angles at vertex of $R$ incident to $f_{2}$ has contribution in $[-8 i-4,-8 i+2]$.

Step (iv) is now completed as follows. Let $e$ be an edge of $P$, and identify the correspond tendril/wiggle $R_{e}$ in $G_{R I}$. Gadget $R_{e}$ is adjacent to two faces $f_{v_{1}}, f_{v_{2}}$ in the dual of $P$ which correspond to the two endpoints $v_{1}, v_{2}$ of $e$ in $P$. If $R_{e}$ makes a positive contribution to $f_{v_{1}}$, then direct edge $e$ towards $v_{1}$, else direct it towards $v_{2}$.

If $R_{e}$ is an RI $i$-tendril, then its contribution is $\approx 8 i$ at the head of $e$ and $\approx-8 i$ at the tail of $e$. Set the flow-value of $e$ to be $i$ (i.e., divide the contribution of $R_{e}$ by 8 and round 
to the nearest integer.) Directly from the definition of $G_{R I}$, this is a permitted capacity for $e$. If $R_{e}$ is an RI $i$-wiggle, then its contribution to the head and tail are approximately $c$ and $-c$ for some $c \leq 8 i$; set the flow-value of $e$ to be the nearest positive integer to $c / 8 \leq i$, which is a permitted capacity.

It remains to verify the balance-equation at each vertex. Since $\Gamma_{R I}$ was an RIdrawing, the sum of labels at a face of degree $k$ is $2 k \pm 4$, hence the contribution of all angles at a face is \pm 4 . Therefore, the contributions of the wiggles and tendrils at these faces must balance out except for $\mathrm{a} \pm 4$. This would prove the balance-equation, except that we have added error by rounding the contributions to the nearest multiple of 8. Now each face has at most $\gamma$ edges (this was the definition of $\gamma$ ), so there are at most $\gamma$ wiggles and tendrils adjacent to the face, so the total error is at most $4 \gamma+4$ after rounding contributions and considering the \pm 4 term. But every capacity in the switch-flow graph is larger than $\theta=4 \gamma+4$ by assumption on the instance. Therefore the balance-equation must hold, since the error adds add most $\theta$.

So from any open planar RI-drawing of $G_{R I}$, we can read a planar embedding of the wiggles and tendrils, and from it directions and capacities for the integer switch-flow problems that satisfy the balance equations. This proves NP-hardness.

\section{Conclusion}

This paper continued the study of [1] and showed how to test for any plane graph whether it has an open planar RI-drawing with non-aligned frame. But if the planar embedding is not fixed, then testing this becomes NP-complete.

The main remaining open problem is: can we drop "with non-aligned frame"? Thus, is there an algorithm to test for any plane graph whether it has an open planar RIdrawing?

\section{References}

1. Alamdari, S., Biedl, T.: Planar Open Rectangle-of-Influence Drawings with Non-aligned Frames. In: van Kreveld, M., Speckmann, B. (eds.) GD 2011. LNCS, vol. 7034, pp. 14-25. Springer, Heidelberg (2012)

2. Battista, G.D., Eades, P., Tamassia, R., Tollis, I.G.: Graph Drawing: Algorithms for the Visualization of Graphs. Prentice Hall (1998), http://www.cs.brown.edu/ rt/gdbook.html

3. Biedl, T., Bretscher, A., Meijer, H.: Rectangle of Influence Drawings of Graphs without Filled 3-Cycles. In: Kratochvíl, J. (ed.) GD 1999. LNCS, vol. 1731, pp. 359-368. Springer, Heidelberg (1999)

4. Biedl, T.C., Kant, G., Kaufmann, M.: On triangulating planar graphs under the fourconnectivity constraint. Algorithmica 19(4), 427-446 (1997)

5. Cornelsen, S., Karrenbauer, A.: Accelerated Bend Minimization. In: van Kreveld, M., Speckmann, B. (eds.) GD 2011. LNCS, vol. 7034, pp. 111-122. Springer, Heidelberg (2012)

6. Cornelsen, S., Schank, T., Wagner, D.: Drawing graphs on two and three lines. J. Graph Algorithms Appl. 8(2), 161-177 (2004)

7. Garg, A., Tamassia, R.: On the computational complexity of upward and rectilinear planarity testing. SIAM J. Comput. 31(2), 601-625 (2001) 
8. Liotta, G., Lubiw, A., Meijer, H., Whitesides, S.: The rectangle of influence drawability problem. Comput. Geom. 10(1), 1-22 (1998)

9. Miura, K., Haga, H., Nishizeki, T.: Inner rectangular drawings of plane graphs. Int. J. Comput. Geometry Appl. 16(2-3), 249-270 (2006)

10. Miura, K., Matsuno, T., Nishizeki, T.: Open rectangle-of-influence drawings of inner triangulated plane graphs. Discrete \& Computational Geometry 41(4), 643-670 (2009)

11. Miura, K., Nishizeki, T.: Rectangle-of-influence drawings of four-connected plane graphs. In: Asia-Pacific Symposium on Information Visualization (APVIS). CRPIT, vol. 45, pp. 7580 (2005)

12. Nishizeki, T., Rahman, M.S.: Planar Graph Drawing. World Scientific (2004)

13. Sadasivam, S., Zhang, H.: Closed rectangle-of-influence drawings for irreducible triangulations. Comput. Geom. Theory Appl. 44(1), 9-19 (2011),

http://dx.doi.org/10.1016/j.comgeo.2010.07.001

14. Tamassia, R.: Handbook of graph drawing and visualization (discrete mathematics and its applications), online draft available at http: / / www.cs.brown. edu/ rt/gdhandbook/

15. Tamassia, R.: On embedding a graph in the grid with the minimum number of bends. SIAM J. Comput. 16(3), 421-444 (1987), http://dx.doi.org/10.1137/0216030

16. Zhang, H., Vaidya, M.: On open rectangle-of-influence and rectangular dual drawings of plane graphs. Discrete Mathematics, Algorithms and Applications 1(3), 319-333 (2009) 